\title{
Erratum to: Value Functions and Transversality Conditions for Infinite-Horizon Optimal Control Problems
}

\section{Nobusumi Sagara}

Published online: 17 March 2010

(C) Springer Science+Business Media B.V. 2010

\section{Erratum to: Set-Valued Anal \\ DOI 10.1007/s11228-009-0132-1}

In the original article an error occurred in Corollary 4.1. The following condition, the uniform coercivity of $L$, is required in addition to the hypothesis in Corollary 4.1.

$$
\lim _{\|x\| \rightarrow \infty} \inf \{L(t, x, u) \mid(t, u) \in \operatorname{graph}(U)\}=\infty .
$$

For completeness, I provide the proof of Corollary 4.1.

Proof of Corollary 4.1 For every admissible process $(x(\cdot), u(\cdot))$ for $(\mathrm{P})$, we have $\lim _{t \rightarrow \infty}\|x(t)\|<\infty$ by $(*)$. Otherwise, $\lim _{t \rightarrow \infty}\|x(t)\|=\infty$ implies that $\lim _{t \rightarrow \infty} L(t$, $x(t), u(t))=\infty$, which obviously contradicts the integrability of $L(\cdot, x(\cdot), u(\cdot))$ over $[0, \infty)$. Hence, $\sup _{t \in[0, \infty)}\|x(t)\|<\infty$. This implies that for every admissible process $(x(\cdot), u(\cdot))$ for $(\mathrm{P})$, there exists some $\varepsilon>0$ such that $x(t) \in x_{0}(t)+\varepsilon B$ for every $t \in[0, \infty)$. By the condition (i) of the corollary, the conditions of Theorem 4.1 are true for $P(t) \equiv 0$. Therefore, we have $J\left(x_{0}(\cdot), u_{0}(\cdot)\right) \leq J(x(\cdot), u(\cdot))$.

(i) In the proof of Lemma 5.1. Delete " $\mu(d u)$ " from the fourth line from the bottom.

(ii) In the second paragraph of Appendix A.3, “ $\Omega(t) \times \mathbb{R}^{n}$ " should read " $\mathbb{R}^{n} \times \mathbb{R}^{n}$ ".

The online version of the original article can be found under http://dx.doi.org/10.1007/s11228-009-0132-1.

N. Sagara $(\bowtie)$

Faculty of Economics, Hosei University 4342, Aihara, Machida, Tokyo, 194-0298, Japan

e-mail: nsagara@hosei.ac.jp 